\title{
The cell lineage of the muscles of the Drosophila head
}

\author{
K. VIJAY RAGHAVAN * AND LUDWIN PINTO \\ Molecular Biology Unit, Tata Institute of Fundamental Research, Homi Bhabha \\ Road, Colaba, Bombay 400005, India
}

\begin{abstract}
SUMMARY
Using a cell marker mutation the cell lineage of the muscles of the Drosophila head are traced out. Three sets of muscles separated by lineage restrictions are observed, even when cells are marked as early as the blastoderm stage. Each set underlies the derivatives of one of the three pairs of imaginal discs which differentiate to form the epidermis of the adult head. Clones of the homoeotic mutation engrailed $\left(e n^{I O}\right)$ were apparently normal in the muscles of the head. The muscle clone frequency, at the blastoderm stage, in each hemisegment of the fly is similar, indicating an equal partitioning of cells during segmentation.
\end{abstract}

\section{INTRODUCTION}

The study of marked clones of cells by genetically labelling their precursors has yielded valuable insights into the nature of development of the insect epidermis (see Lawrence, 1981a, for a recent review). It is relevant to ask whether the principles and hypotheses that have resulted from these studies have any general value in understanding how complex organisms are constructed. With this in mind Lawrence and his colleagues (Lawrence, 1982; Lawrence \& Brower, 1982; Lawrence \& Johnston, 1982) have studied the development of the muscles of Drosophila and have traced out the cell lineage of the adult thorax and abdomen. We report here the cell lineage of the muscles of the head of the adult fly and test the role of engrailed ${ }^{I O}$ (Kornberg, 1981a) in these muscles.

Our results suggest that the precursors of the muscles of the head and the precursors of the epidermis of the head, though of separate clonal origins, are partioned together early in development in a specific manner.

\section{MATERIALS AND METHODS}

\section{Preparation of muscle whole mounts}

Dissected preparations of thoracic, abdominal or cephalic muscles were visualized in whole mounts. Heads or thoraces were dissected in Ringer's solution stained for succinate dehydrogenase $(s d h)$ activity as described below, fixed for $15 \mathrm{~min}$ in ammoniacal alcohol, dehydrated in absolute alcohol, cleared in methyl salicylate for 10-30 min (it is important not to exceed this time) and mounted in Struhl's mounting medium (Struhl, 1981a).

*Present address: Division of Biology, California Institute of Technology, Pasadena, California, 91125, USA.

Key words: Drosophila, cell lineage, muscles, engrailed $\left(e n^{I O}\right)$. 


\section{Clonal analysis: generating mitotic clones by $X$-irradiation}

The cell lineage of the muscles of the Drosophila head was traced by inducing clones of cells homozygous for $s d h^{8}$ (Lawrence, 1981b), a mutation which affects the activity of succinate dehydrogenase. X-irradiation of dividing cells of the genotype $s d h^{8} M(2) c^{+} / s d h^{+} M(2) c^{33 a}$ can yield, by appropriate mitotic recombination, a daughter cell homozygous for $s d h^{8}$. Cells of the genotype $s d h^{8} M(2) c^{+} / s d h^{8} M(2)^{+}$, thus generated, will compete with and have a growth advantage over cells of the parental genotype $s d h^{8} M(2) c^{+} / s d h^{+} M(2) c^{33 a}$ (The 'Minute' technique, Morata \& Ripoll, 1975). Some of the $M(2) c^{+}$cells will thus demarcate the boundaries of lineage restrictions, if they exist. $c n s d h^{8} b w / C y O$ females were crossed to $c n$ $b w M(2) c^{33 a} / b w^{\mathrm{V} 32 \mathrm{~g}}$ males. Eggs were collected in carefully timed batches and irradiated at various times during development (Lawrence, 1982). $c n b w M(2) c^{33 a} / c n s d h^{8} b w$ progeny from the above cross were collected. Their heads were cut away from the thoraces, dissected open and stained overnight in $s d h$ staining solution (Lawrence, 1981b) after incubation in Ringer's solution for $45 \mathrm{~min}$ at $48^{\circ} \mathrm{C}$. The procedure for staining the thoraces and abdomens were as described in Lawrence (1982) and Lawrence \& Johnston (1982), respectively. In some cases the epidermis too was genetically marked. The markers used for this purpose were straw (stw), pawn (pwn) and sdh (Lawrence \& Johnston, 1982).

\section{Visualization of clones}

Patches of unstained fibres are visible clearly in tissues which have clones in them. Stained fibres are dark blue, while unstained fibres are pale blue or white. When polarized light optics are used the unstained fibres stand out brightly in sharp contrast to the stained fibres.

\section{Clones of engrailed}

The role of the homoeotic gene engrailed in the cephalic musculature was tested. This was done by examining the frequency and nature of $e n^{I O} s d h^{8}$ clones and comparing them with $e n^{+}$ $s d h^{8}$ clones in the muscles. The locus for engrailed is at 2-64 (Kornberg, 1981a) and that for $s d h$ at 2-89. Therefore, in the X-ray-induced mitotic recombinants generated in the experiment to look at $e n^{I O}$ clones, some of the $s d h^{8}$ clones will be $e n^{+}$. It is therefore necessary to examine the data quantitatively (Lawrence, 1982). The following crosses were performed in order to do this: $c n s d h^{8} b w / c n M(2) c^{33 a} b w$ males were crossed to $s t w p w n e n^{+} c n s d h^{8} b w / C y O$ females as controls and to $s t w$ pwn en ${ }^{I O} s d h^{8} b w s p / C y O$ as experimentals. The clone frequency amongst the progeny of control and experimental animals (grown together in the same bottles under identical conditions) were compared. All progeny from the above crosses were irradiated $48 \pm 4 \mathrm{~h}$ after egg laying, the X-ray dose being $1500 \mathrm{R}$.

\section{Mutant stocks}

All mutant stocks were kindly provided by Dr P. A. Lawrence and have been described in Lawrence (1982), and in Lawrence \& Johnston (1982).

\section{RESULTS}

The muscles of the Drosophila head have been described by Miller (1950) and are illustrated in Fig. 1. There are about 16 muscles in each half of the adult head.

Using a cell marker mutation that affects the activity of succinate dehydro genase (Lawrence, 1981b), muscle precursors were genetically marked by X-rayinduced mitotic recombination. The 'Minute' technique was used, giving the marked cells a growth advantage. Under the conditions described the marked cells were pale blue or completely white, while the background was dark blue. 

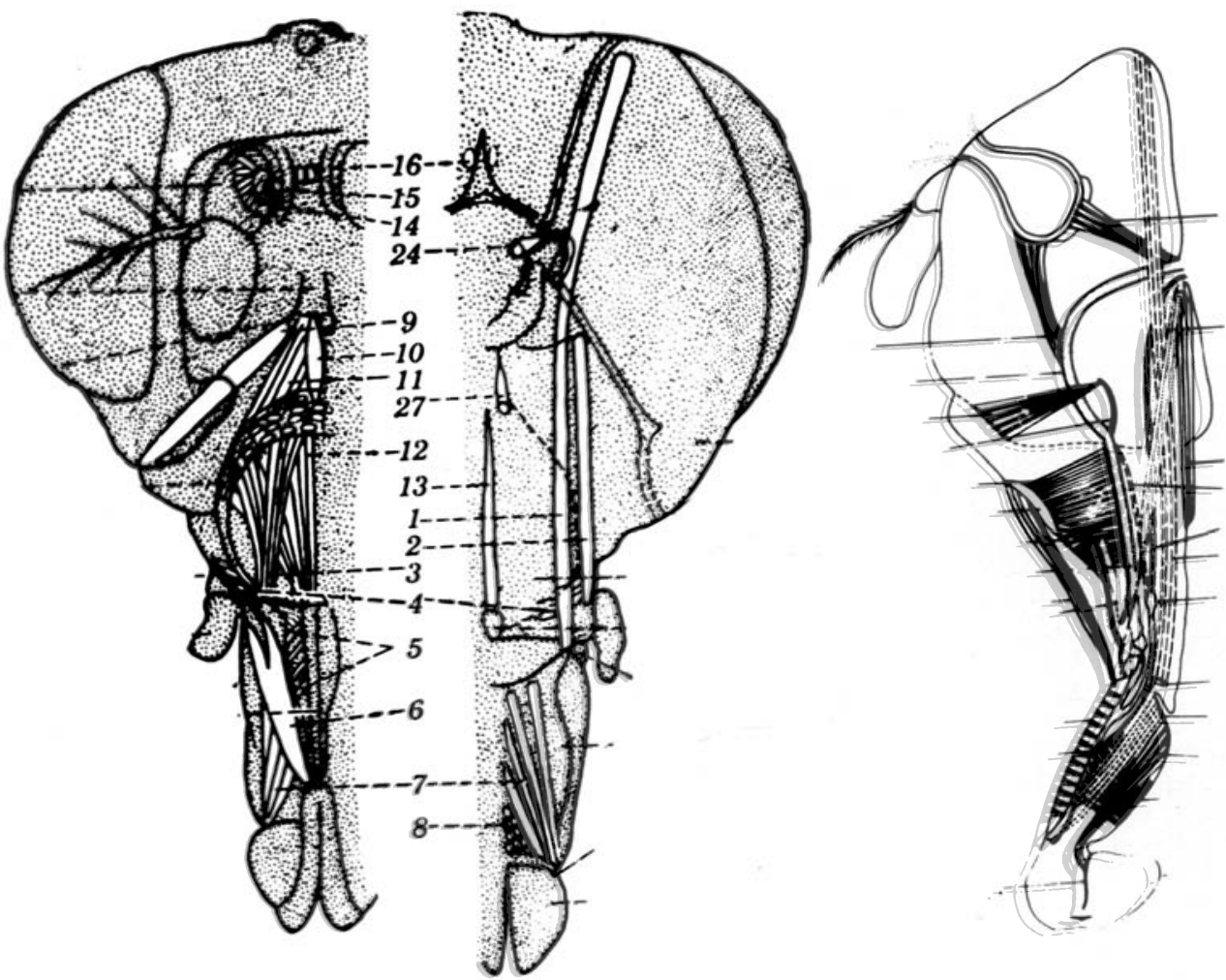

Fig. 1. The muscles of the Drosophila head. (A) A frontal view, adapted from Miller (1950). (B) A saggital view, adapted from Crossley (1978).

Cell lineage of the cephalic muscles: description of clones following irradiation of larvae

Larvae were irradiated at different stages during development: $36 \pm 2$ (mid first instar), $48 \pm 4$ (late first instar) and $72 \pm 6 \mathrm{~h}$ (late second instar) after egg laying. The data presented are for $48 \pm 4 \mathrm{~h}$ after egg laying as this time of irradiation yielded a convenient clone size and frequency. The results are qualitatively the same for all the stages studied. The principal result is that the clones fell into three sets on the basis of lineage restrictions: clones in one set did not overlap with clones in the other two sets. Further, clones never left a set completely unstained. The muscle fibres which constitute each set, as defined by the lineage restrictions, are stated below.

Set I

Clones in this set marked the fibres in muscle 9, Fig. 1 (the total number of clones observed for each set and the clone frequency are outlined in Table 1). 


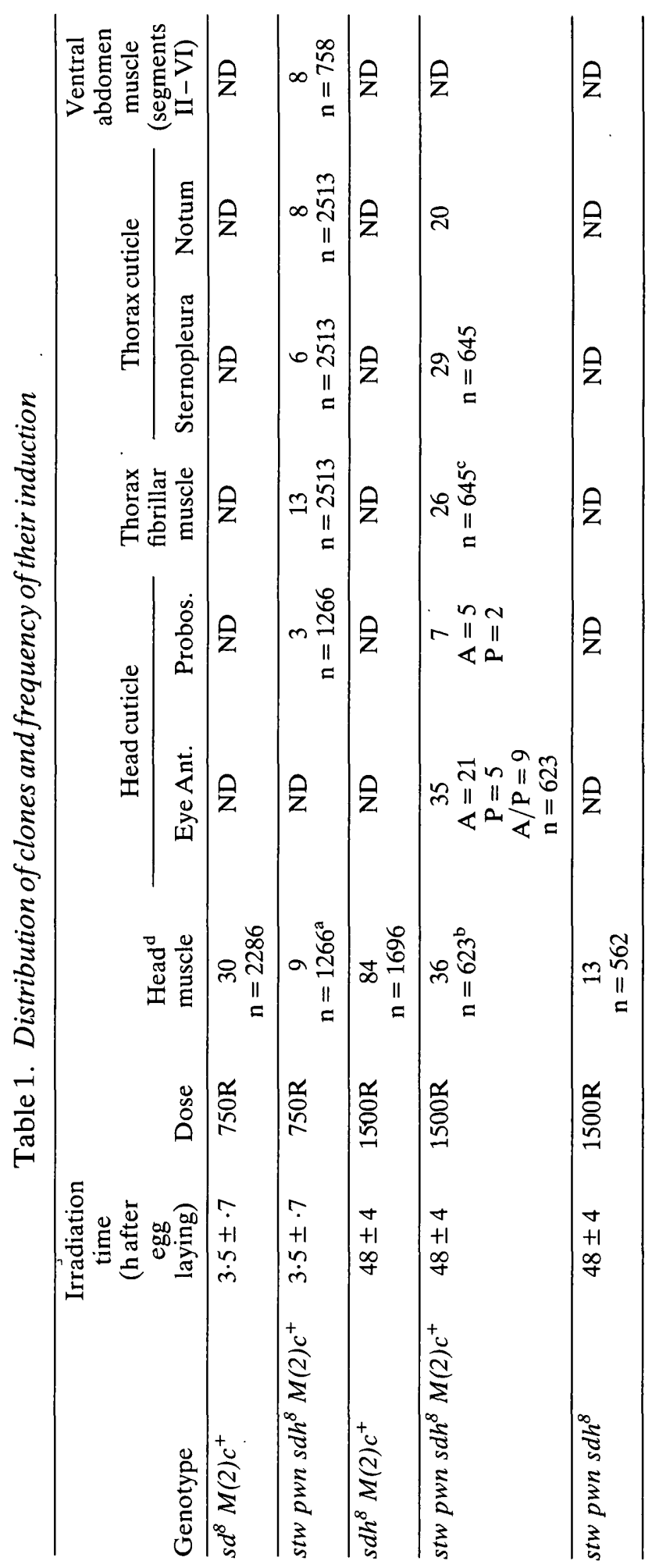


Clones in Set I were always unilateral. Clones were never observed to cross to other nearby muscles (for example, muscles 11, 12, 3 and 4; Fig. 1). Fig. 2 shows a clone in muscle Set $\mathrm{I}$.

\section{Set II}

Clones in muscles 3, 4, 11 and 12 (Fig. 1) cross freely between one another but not to other muscles. These muscles were allotted to Set II. From the data on the frequency and distribution of the clones in this set (Table 1) it can be seen that a large fraction of the clones were bilateral. Fig. 2 shows a clone in Set II.

\section{Set III}

Clones in Set III were restricted to muscles 5, 6, 7 and 8 (Fig. 1) and did not cross to other muscles. The data (Table 1 ) shows that a large number of the clones were bilateral. Fig. 2 shows a clone in Set III.

\section{Description of clones following irradiation at the blastoderm stage}

The frequency of clones following irradiation at the blastoderm stage $(3 \cdot 5 \pm 7 \mathrm{~h}$ after egg laying) was low, but significantly different from unirradiated controls (Table 1). The pattern of clones was the same as that for the later stages of

Table 1. Footnotes

$\mathrm{ND}=$ not done

$\mathrm{n}=$ total number of samples

$\mathrm{A}=$ Anterior

$\mathrm{P}=$ Posterior

${ }^{a}$ Of the 1266 heads, three clones were observed in the proboscis. No muscle clones were observed in the muscles underlying the proboscis in these three heads. In the remaining heads nine muscle clones were observed.

${ }^{\mathrm{b}}$ Of the 623 heads 35 clones were observed in the eye-antenna cuticle and 7 in the proboscis. No muscle clones were observed in preparations bearing cuticular clones. 33 muscle clones were observed in the remaining preparations.

${ }^{c}$ Of the 645 thoraces 29 clones were detected in the sternopleura and 20 in the notum. None of these clones bearing thoraces had muscle clones in fibrillar muscle except in one case where a clone was observed in the contralateral side. 25 fibrillar muscle clones were detected in the remaining thoraces.

${ }^{\mathrm{d}} \mathrm{A}$ total of 130 muscle clones in 2881 heads were detected after larval irradiations. 44 underlying the eye antenna disc derivatives, 54 underlying the derivatives of the cibarial disc and 32 underlying the derivatives of the labial disc. 19 of the 54 clones underlying the cibarial disc derivatives were bilateral. Nine of the 32 clones underlying the derivatives of the labial disc were bilateral. This could possibly be because these discs are close together during development and the use of the Minute technique allows marked cells to cross from one side to another.

${ }^{\mathrm{e}} \mathrm{A}$ total of 39 muscle clones in 3552 heads were detected after blastoderm radiation. 15 underlying the eye-antenna disc derivatives, 17 (four bilateral) underlying the cibarial disc derivatives and 7 (two bilateral) underlying the derivatives of the labial disc.

No putative clones were observed in 3500 unirradiated controls. None were observed in 1250 irradiated $(48 \pm 4 \mathrm{~h})$ controls. The genotype of the unirradiated controls were the same as that of the experimental animals. The genotype of the irradiated controls were $c n s d h^{+} b w$ $M(2) c^{33 a} / \mathrm{CyO} s d h^{+}$. 
development; no case of violation of the above three sets of lineage restrictions were observed (39 clones scored).

\section{Muscles not allotted to any set}

The following muscles could not be allotted to any of the three sets. Muscles 1, 2, 14, 15, 16 and 24 (Fig. 1) were damaged during preparation of the samples and possible clones in them were therefore disregarded. Muscles 27 and 10 are very small and are obscured by nearby muscles. While large clones in Set II sometimes seemed to encompass these muscles, a categorical allotment could not be made.

\section{The role of engrailed in the musculature}

The engrailed gene has been shown to be required exclusively in the posterior compartment of the adult epidermis (Morata \& Lawrence, 1975, 1979; Lawrence \& Morata, 1977; Lawrence, Struhl \& Morata, 1979; Kornberg, 1981a,b; Lawrence \& Struhl, 1982). Mutations at this locus affect the anterior-posterior compartition and clones, normally restricted, can cross from the posterior to anterior. Are the muscles, like the epidermis, also divided into anterior and posterior compartments? This question has been put to test in the muscles of the thorax and abdomen of the adult fly (Lawrence, 1982; Lawrence \& Johnston, 1982). We have tested the role of $e n^{I O}$, a lethal allele of engrailed (Kornberg, 1981a), in the muscles of the head. The results are presented in Table 2. Both the frequency and the phenotype of the clones were no different from that observed by marking the muscles only with $s d h$. This result is similar to that observed for the other muscles of the fly (Lawrence, 1982; Lawrence \& Johnston, 1982).

\section{The frequency of clones in the muscles}

Clone frequency at different stages of development can give crude estimates of the number of precursors present. Many assumptions are implicit in such calculations and the results are therefore limited in their value (Lawrence \& Johnston, 1982). The data used to calculate the frequencies of clones in different muscle sets and developmental stages are presented in Table 1. According to the study of Struhl (1981b), of the six segments that constitute the head of Drosophila three contribute to the adult head and the other three probably contribute only to

Fig. 2. (A,B,C,D) Clone in set I. Frontal view. (A) Shows a preparation of the head under bright-field illumination and (B) shows the clone (arrows) which can be visualized under polarized light optics. (C) Shows this clone at higher magnification under bright-field illumination and (D) under phase-contrast optics where the unstained muscles (arrows) can be seen. (E) Shows a saggital view of a head preparation under bright-field illumination and $(F)$ under polarized light optics where the clone in set II (arrows) can be seen. (G,H,I) Clone in set III. Frontal view. The clone is bilateral, $(\mathrm{G})$ bright-field illumination, $(\mathrm{H})$ polarized light optics (arrows show an unstained muscle in focus). (I) The clone in higher magnification showing stained nerves (arrow) going to unstained muscles. Bar $=0.05 \mathrm{~mm}$. 

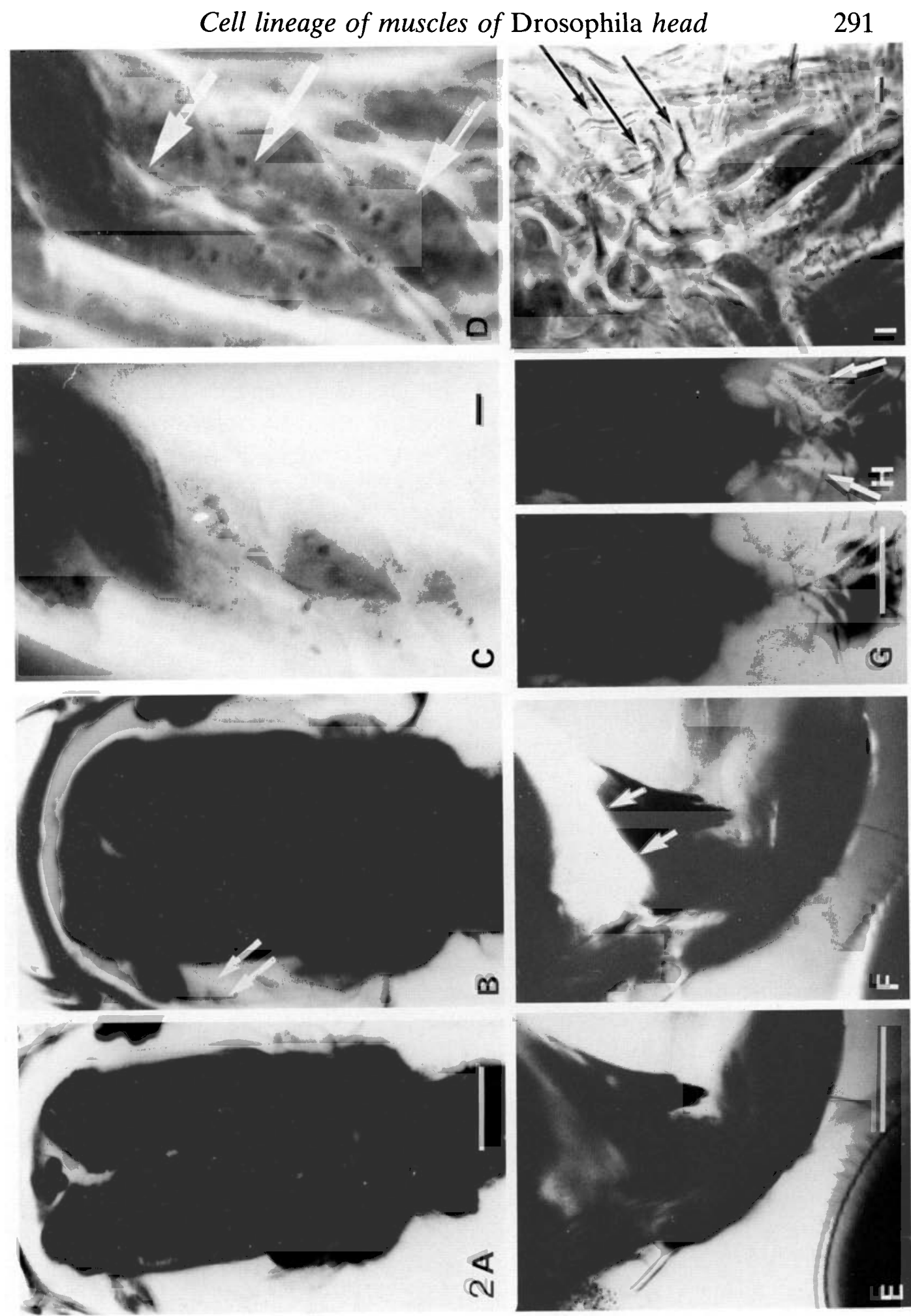
Table 2. Frequency of $\mathrm{en}^{\mathrm{IO}}$ clones. Radiation at $48 \pm 4 \mathrm{~h}$ after egg laying, $1500 \mathrm{R}$

\begin{tabular}{lc}
\hline Genotype & Clones in head \\
\hline$e n^{+} s d h^{8} M(2) c^{+}$ & 14 \\
$e n^{I O} s d h^{8} M(2) c^{+}$ & $\mathrm{n}=562$ \\
& 18 \\
& $\mathrm{n}=495$
\end{tabular}

$\mathrm{n}=$ total number of samples.

larval structures. The three segments that constitute the structures of the adult epidermis are, according to the analysis of sturt distances (Struhl, 1981b), the derivatives of the eye-antenna disc, the cibarial disc and the labial disc. Our results for the muscles of the head show three sets separated by lineage restrictions, each set falling under the derivatives of one of the three imaginal discs that differentiate to form the cephalic epidermis. Assuming that each set of muscles underlies a segment of the epidermis, the clone frequency per hemisegment can be calculated.

The clone frequency at the blastoderm stage for the muscles of the head is about 2/1000 hemisegments (there are six hemisegments in the head and a total of 39 blastoderm clones were observed in 3552 heads scored; Table 1). A similar calculation for the muscles of the thorax (Lawrence, 1982) and the abdomen (Lawrence \& Johnston, 1982) yielded a frequency of $1 / 1000$ hemisegments.

At the larval stage ( $48 \pm 4 \mathrm{~h}$ after egg laying) the clone frequency in the muscles of the head becomes $8 / 1000$ hemisegments. Allowing for the differences in radiation dose between this stage and the blastoderm stage, the clone frequency is consistent with about two cell divisions having taken place since the blastoderm stage.

\section{Dose dependance of clones}

That the clones were genuine and not artifacts of staining was ascertained by observing that when the muscles were unstained the nerves to these fibres and the surrounding epidermis were stained. The frequency of clones increases with later irradiations. These results show that the clones are not artifacts of radiation or staining (Lawrence, 1982).

\section{The cephalic muscles are of mesodermal origin}

Clones were never observed to cross between the epidermis and the underlying. musculature. This is so even when the precursors are marked at the blastoderm stage (see Table 1; footnotes $\mathrm{a}, \mathrm{b}$ and $\mathrm{c}$ ). There is therefore a lineage restriction between the muscles and the epidermis. At least some of the precursors of the muscles are known to be the adepithelial cells which can be identified on the imaginal discs at the third instar larval stage (Lawrence \& Brower, 1982). The above results show that the adepithelial cells, though attached to the imaginal 
discs, are not derived from epidermal precursors and are probably of mesodermal origin.

\section{DISCUSSION}

The results presented here give the following picture of the development of the head of the adult fly. At segmentation, equal numbers of precursors of the muscles and the epidermis are probably partioned into each metameric unit of the animal. This conclusion is drawn from the similarity of clone frequency at the blastoderm stage in each hemisegment (Table 1; Lawrence \& Johnston, 1982). Even at the blastoderm stage the muscles of the head derive from three sets of precursors. Each set develops to form muscles which underly the derivatives of an imaginal disc. Each of the derivatives of the eye-antennal disc, the cibarial disc and the labial disc constitutes a compartment since clones do not cross from one imaginal disc derivative to the others. Addressing himself to the question of the number of segments that constitute the insect head, Struhl (1981b) argued that the insect head was segmented and further, was made up of six segments. Three segments contributed to the adult head structures and six to larval structures. The three lineage sets observed in the muscles of the head are compartments, by definition. The number and location of these sets suggest that the mesoderm is also segmented in a manner similar to that of the cephalic epidermis. As mentioned earlier some muscles could not be allotted to any of the sets. It is therefore not possible to say that all the muscles of the head belong to one of the three sets that were observed. Some of these muscles may be derived from precursors that may contribute to larval muscles too.

Do the muscle sets have a genetic basis? To test this, clones of a lethal allele of engrailed, $e n^{I O}$, were examined in the muscles of the head. These clones were observed at a normal frequency and did not seem any different from the $\mathrm{en}^{+}$ clones. The normal phenotype and frequency of $e n^{I O}$ clones do not of course rule out the possibility that other alleles of engrailed may have a detectable phenotype in the muscles. But the result certainly suggests that homoeotic genes like engrailed may not act in the muscles. Their role may be restricted to the epidermis and may be other ectodermal derivatives. This would suggest that the epidermis of the fly is rigidly programmed but the muscles probably develop according to inductive instructions from the epidermis (Lawrence, 1982). This view of muscle development in Drosophila is too simple - recent results indicate that the homoeotic gene Ultrabithorax is functional in the muscles of the abdomen (Akam, 1983; Lawrence \& Johnston, 1984). Our study was restricted to only one allele of engrailed. Studies on combinations of this mutation which affect head cuticle, $\left(e n^{1} / e n^{c 2}\right.$, Morata, Kornberg \& Lawrence, 1983) may reveal effects on the muscles of the head.

The work was begun at the MRC Laboratory of Molecular Biology, Cambridge, England, where KVR was a visitor supported by the British Council. We thank Peter Lawrence 
for his help and advice. Susan Mangrum's efforts in typing the manuscript are gratefully acknowledged.

\section{REFERENCES}

Aкам, M. E. (1983). The location of Ultrabithorax transcripts in Drosophila tissue sections. The EMBO Journal 2, 2075-2084.

Crossley, A. C. (1978). The Genetics and Biology of Drosophila (eds. M. Ashburner \& T. R. F. Wright), vol. 2b. London: Academic Press.

KoRNBERG, T. (1981a). Engrailed: a gene controlling compartment and segment formation in Drosophila. Proc. natn. Acad. Sci., U.S.A. 78, 1095-1099.

Kornberg, T. $(1981 b)$. Compartments in the abdomen of Drosophila and the role of the engrailed locus. Devl Biol. 86, 363-381.

LAWRENCE, P. A. (1981a). The cellular basis of segmentation in insects. Cell 26, 3-10.

LAWRENCE, P. A. (1981b). A general cell marker for clonal analysis of Drosophila development. J. Embryol. exp. Morph. 64, 321-332.

LAWRENCE, P. A. (1982). Cell lineage of the thoracic muscles of Drosophila. Cell 29, 493-503.

LAWrence, P. A. \& Brower, D. L. (1982). Myoblasts from Drosophila wing disks can contribute to developing muscles throughout the fly. Nature 295, 55-57.

Lawrence, P. A. \& Morata, G. (1977). The early development of the mesothoracic compartments of Drosophila. Devl Biol. 56, 40-51.

Lawrence, P. A., Struhl, G. \& Morata, G. (1979). Bristle patterns and compartment boundaries in the tarsus of Drosophila. J. Embryol. exp. Morph. 51, 195-208.

LaWrence, P. A \& Johnston, P. (1982). Cell lineage of Drosophila abdomen: The epidermis, the oenocytes and the ventral muscles. J. Embryol. exp. Morph. 72, 197-208.

Lawrence, P. A. \& Johnston, P. (1984). The genetic specification of pattern in a Drosophila abdomen. Cell 36, 775-782.

Lawrence, P. A. \& Struhl, G. (1982). Further studies on the engrailed phenotype in Drosophila. The EMBO Journal 1, 827-833.

Lindsley, D. L. \& Grell, E. H. (1968). Genetic variations in Drosophila melanogaster. Carnegie Inst. Wash. Publ. No. 627.

Miller, A. (1950). The Biology of Drosophila (ed. M. Demerec), pp. 420-554. New York: Wiley.

Morata, G. \& Lawrence, P. A. (1975). Control of compartment development by the engrailed gene in Drosophila. Nature 255, 614-617.

Morata, G. \& Lawrence, P. A. (1977). Homeotic genes, compartments and cell determination in Drosophila. Nature 265, 211-216.

Morata; G. \& Lawrence, P. A. (1979). Development of the eye-antenna imaginal disc of Drosophila. Devl Biol. 70, 355-371.

Morata, G. \& Ripoll, P. (1975). Minutes: mutants of Drosophila affecting cell division rate. Devl Biol. 42, 211-221.

Morata, G., Kornberg, T. \& Lawrence, P. A. (1983). The phenotype of engrailed mutations in the antenna of Drosophila. Devl Biol. 99, 27-33.

Struhl, G. (1981a). Anterior and posterior compartments in the proboscis of Drosophila. Devl Biol. 84, 372-385.

STruHL, G. (1981b). A blastoderm fate map of compartments and segments of the Drosophila head. Devl Biol. 84, 386-396. 